\title{
Efficiency of shock capturing schemes for Burgers' equation with boundary uncertainty
}

\author{
Per Pettersson $^{a, b, 1}$, Qaisar Abbas ${ }^{b, 2}$, Gianluca Iaccarino $^{*, 3}$ and Jan Nordström $^{b, c, d, 4}$ \\ ${ }^{a}$ Department of Mechanical Engineering \\ Stanford University, 488 Escondido Mall, Stanford, 94305, CA, USA \\ ${ }^{1}$ massperp@stanford.edu, ${ }^{3}$ jops@ @ stanford.edu. \\ ${ }^{b}$ Department of Information Technology \\ Uppsala University, SE-751 05 Uppsala, Sweden \\ ${ }^{2}$ qaisar.abbas@it.uu.se, ${ }^{4}$ jan.nordstrom@it.uu.se. \\ ${ }^{c}$ School of Mechanical, Industrial and Aeronautical Engineering \\ University of the Witvatersrand, PO WITS 2050, Johannesburg, South Africa \\ ${ }^{d}$ Department of Aeronautics and Systems Integration \\ FOI, The Swedish Defense Research Agency, SE-164 90 Stockholm, Sweden
}

Keywords: Uncertainty quantification, hyperbolic systems, high resolution schemes

\begin{abstract}
Burgers' equation with uncertain initial and boundary conditions is approximated using a polynomial chaos expansion approach where the solution is represented as a series of stochastic, orthogonal polynomials. Even though the analytical solution is smooth, a number of discontinuities emerge in the truncated system. The solution is highly sensitive to the propagation speed of these discontinuities. High-resolution schemes are needed to accurately capture the behavior of the solution. The emergence of different scales of the chaos modes require dissipation operators to yield accurate solutions. We will compare the results using the MUSCL scheme with previously obtained results using conventional one-sided operators.
\end{abstract}

\section{Introduction}

The inviscid Burgers' equation is investigated subject to uncertain boundary and initial conditions. The stochastic solution is represented as a polynomial chaos series, using a suitable basis of orthogonal stochastic polynomials. The stochastic Galerkin method [1] is applied and the stochastic equation is projected onto a stochastic polynomial basis yielding a system of deterministic equations for the time and space dependent coefficients of the series. The resulting system is hyperbolic and exhibit multiple discontinuities in finite time. This motivates the use of high-resolution schemes.

We investigate two different approaches. First, a central scheme with local artificial dissipation is investigated. Summation by parts operators (SBP) [2] and the simultaneous approximation term (SAT) technique [3] to impose boundary conditions weakly lead to stability. The amount 
of artificial dissipation should be proportional to the system eigenvalues which are generally unknown a priori. Secondly, we study the monotone upstream centered schemes for conservation laws (MUSCL) approach originally developed by van Leer [4]. We use the minmod limiter and Roe averages to approximate the fluxes, see [5].

Both types of schemes exhibit excellent properties of shock capturing for model problems such as the scalar Burgers' equation. However, the hyperbolic systems resulting from the stochastic Galerkin projection are considerably more demanding in several ways. The non-linearity of the problem results in poor convergence properties independent of the numerical method. Also, finer grids are needed for convergence of higher order polynomial chaos systems, increasing the computational cost.

The paper is organized as follows. The systems of equations is derived in section 2 , followed by an outline of the numerical methods in section 3. Section 4 contains numerical experiments where the efficiency of the numerical methods are investigated. Finally, section 5 contains a discussion and concluding remarks.

\section{Polynomial chaos approximation of Burgers' equation}

The polynomial chaos representation of the solution $u(x, t, \xi)=\sum_{i=0}^{\infty} u_{i}(x, t) \Psi_{i}(\xi)$ is inserted into the inviscid Burgers' equation,

$$
u_{t}+u u_{x}=0,0 \leq x \leq 1
$$

which yields

$$
\sum_{i=0}^{\infty} \frac{\partial u_{i}}{\partial t} \Psi_{i}(\xi)+\left(\sum_{j=0}^{\infty} u_{j} \Psi_{j}(\xi)\right)\left(\sum_{i=0}^{\infty} \frac{\partial u_{i}}{\partial x} \Psi_{i}(\xi)\right)=0 .
$$

A stochastic Galerkin projection is performed by truncating the polynomial chaos expansion to order $M$, multiplying (2) by $\Psi_{k}(\xi)$ for non-negative integers $k$ and integrating over the probability domain. The orthogonality of the basis polynomials $\left(\Psi_{i}\right)$ then yields a system of deterministic equations. The result is a symmetric system of deterministic equations,

$$
\frac{\partial u_{k}}{\partial t}\left\langle\Psi_{k}^{2}\right\rangle+\sum_{i=0}^{M} \sum_{j=0}^{M} u_{i} \frac{\partial u_{j}}{\partial x}\left\langle\Psi_{i} \Psi_{j} \Psi_{k}\right\rangle=0 \quad \text { for } k=0,1, \ldots, M
$$

To simplify notation, equation (3) can be written in conservative matrix form as

$$
B u_{t}+\frac{1}{2} \frac{\partial}{\partial x}(A(u) u)=0
$$

where $(B)_{j k}=\delta_{j k}\left\langle\Psi_{j}^{2}\right\rangle$ and $(A(u))_{j k}=\sum_{i=0}^{M} u_{i}\left\langle\Psi_{i} \Psi_{j} \Psi_{k}\right\rangle$. The polynomial basis is chosen to be the set of Hermite polynomials.

We consider the Riemann problem

$$
u(x, t=0, \xi)= \begin{cases}1+\hat{\sigma} \xi & \text { for } x<0.5 \\ -1+\hat{\sigma} & \text { for } x>0.5\end{cases}
$$

and use the time dependent analytical solution for the Cauchy problem derived in [6] with $\hat{\sigma}=0.1$. 


\section{Numerical methods}

\subsection{Central differences}

We approximate the first derivative with a matrix operator of the form $P^{-1} Q$ where $P$ is a positive diagonal matrix and $Q$ satisfies $Q+Q^{T}=\operatorname{diag}(-1,0, \ldots, 0,1)$. For a more detailed description of this technique, see [7], [8].

The system (4) is semi-discretized as

$$
(I \otimes B) u_{t}+\frac{1}{2}\left(P^{-1} Q \otimes I\right) A_{g} u=\left(P^{-1} \otimes I\right)\left[\left(E_{0} \otimes \Sigma_{0}\right)\left(u-g_{0}\right)+\left(E_{n} \otimes \Sigma_{1}\right)\left(u-g_{1}\right)\right] .
$$

A split approach is used to show stability [9] and artificial dissipation [6] is added in the form

$$
A_{2 k}=-\Delta x P^{-1} \tilde{D}_{k}^{T} B_{w} \tilde{D}_{k},
$$

where $\tilde{D}_{k}$ is an approximation of $(\Delta x)^{k} \partial^{k} / \partial x^{k}$ and $B_{w}$ is a diagonal positive definite matrix.

\subsection{MUSCL scheme}

The semi-discrete system is given by

$$
\left(u_{t}\right)_{i}+\frac{F_{i+\frac{1}{2}}-F_{i-\frac{1}{2}}}{\Delta x}=0
$$

where

$$
F_{i+\frac{1}{2}}=\frac{1}{2}\left(F\left(u_{i+\frac{1}{2}}^{L}\right)+F\left(u_{i+\frac{1}{2}}^{R}\right)\right)+\frac{1}{2}\left|A_{i+\frac{1}{2}}\right|\left(u_{i+\frac{1}{2}}^{L}-u_{i+\frac{1}{2}}^{R}\right)
$$

with the absolute value of the Roe average $A_{i+\frac{1}{2}}$ given by

$$
\left|A_{i+\frac{1}{2}}\right|=X\left|\Lambda\left(u_{i+\frac{1}{2}}\right)\right| X^{-1}=\frac{1}{2} X\left|\Lambda\left(u_{i+\frac{1}{2}}^{L}\right)+\Lambda\left(u_{i+\frac{1}{2}}^{R}\right)\right| X^{-1} .
$$

where $\Lambda(u)$ is a diagonal matrix with the eigenvalues of $A(u)$ and $X$ is the eigenvector matrix. The left and right states are given by

$$
u_{i+\frac{1}{2}}^{L}=u_{i}+0.5 \phi\left(r_{i}\right)\left(u_{i+1}-u_{i}\right) \quad \text { and } \quad u_{i+\frac{1}{2}}^{R}=u_{i+1}-0.5 \phi\left(r_{i+1}\right)\left(u_{i+2}-u_{i+1}\right)
$$

respectively. The flux limiter $\phi(r)$ is the minmod limiter. For a more detailed description of the MUSCL scheme, see e.g. [5].

\section{Numerical experiments}

The true solution of the original problem (2) is qualitatively different from the solution of any truncated system after stochastic Galerkin projection. A fair measure of the efficiency of the numerical method should take this into account. The reference solution used for the first order polynomial chaos is therefore the analytical solution to the system (3) with $M=1$. The discrete error norm used for the coefficient $u_{i}$ is given by

$$
\left\|\epsilon_{u_{i}}\right\|_{h}=\sqrt{\frac{\sum_{j=1}^{m}\left(\left(u_{i}^{\text {num }}\right)_{j}-\left(u_{i}^{r e f}\right)_{j}\right)^{2}}{m-1} .}
$$


Table 1: Grid convergence, $M=1, T=0.3$.

\begin{tabular}{lllll}
\hline & Cent./SBP & \multicolumn{3}{c}{ MUSCL } \\
$\mathrm{m}$ & $\left\|\epsilon_{u_{0}}\right\|_{h}$ & $\left\|\epsilon_{u_{1}}\right\|_{h}$ & $\left\|\epsilon_{u_{0}}\right\|_{h}$ & $\left\|\epsilon_{u_{1}}\right\|_{h}$ \\
\hline 51 & 0.1207 & 0.1871 & 0.1309 & 0.2100 \\
101 & 0.0856 & 0.1330 & 0.0934 & 0.1498 \\
201 & 0.0607 & 0.0943 & 0.0664 & 0.1067 \\
401 & 0.0430 & 0.0667 & 0.0479 & 0.0764 \\
801 & 0.0304 & 0.0472 & 0.0358 & 0.0554 \\
\hline
\end{tabular}

Table 2: Grid convergence, $M=3, T=0.3$.

\begin{tabular}{lllllllll}
\hline \multicolumn{1}{c}{ Cent./SBP } & \multicolumn{7}{c}{ MUSCL } \\
$\mathrm{m}$ & $\left\|\epsilon_{u_{0}}\right\|_{h}$ & $\left\|\epsilon_{u_{1}}\right\|_{h}$ & $\left\|\epsilon_{u_{2}}\right\| \|_{h}$ & $\left\|\epsilon_{u_{3}}\right\|_{h}$ & $\left\|\epsilon_{u_{0}}\right\|_{h}$ & $\left\|\epsilon_{u_{1}}\right\|_{h}$ & $\left\|\epsilon_{u_{2}}\right\|_{h}$ & $\left\|\epsilon_{u_{3}}\right\|_{h}$ \\
\hline 51 & 0.0928 & 0.1001 & 0.1487 & 0.0557 & 0.0899 & 0.0950 & 0.1291 & 0.0569 \\
101 & 0.0766 & 0.0740 & 0.1259 & 0.0510 & 0.0652 & 0.0754 & 0.0819 & 0.0336 \\
201 & 0.0594 & 0.0551 & 0.0980 & 0.0423 & 0.0399 & 0.0469 & 0.0566 & 0.0240 \\
401 & 0.0371 & 0.0318 & 0.0626 & 0.0281 & 0.0183 & 0.0157 & 0.0292 & 0.0128 \\
\hline
\end{tabular}

Tables 1-2 show the grid convergence up to third order polynomial chaos. For expansions of higher order $(M>1)$, a solution on a fine mesh $(m=800)$ is used as reference solution, since the analytical solution to the truncated system is unknown.

The number of shocks increase with the order of polynomial chaos, and requires a finer spatial mesh for convergence. As the order of polynomial chaos is increased, we expect a more accurate approximation to the original problem, before truncation of the polynomial chaos expansion. However, as shown in Table 3, the convergence is not monotone. Also, the computational cost strongly increases with the order of polynomial chaos. Accordingly, high order expansions are not necessarily desirable for these problems.

In order to understand and remedy these issues, consider the coarse grid solution of Figure 1, where the SBP solution with artificial dissipation appears to be a more accurate approximation of the true analytical solution than the MUSCL solution. By scaling the $i^{t h}$ Hermite polynomial by $1 / \sqrt{i !}$ of the MUSCL scheme, the solution approaches the SBP solution on an equal grid, Figure 2. However, unlike the scalar cases and lowest order of polynomial chaos, this solution is not grid converged. Since these numerical solutions are solutions to the truncated system, we can not evaluate the efficiency of the numerical methods by comparison with the true analytical solutions only.

With $m=400$ mesh points, the solutions are grid converged, but different due to the nonsharp eigenvalue estimate of the SBP approach which modifies the artificial dissipation and the solution, Figure 3. By successively decreasing the amount of artificial dissipation to the point where the solution fails to converge, the SBP solution approaches the MUSCL solution, Figure 4. The two different scalings of the basis polynomials also result in the same solution as the mesh is refined (Figure 5). 
Table 3: PC expansion convergence, $m=100, T=0.3$.

\begin{tabular}{lllll}
\hline & Cent./SBP & \multicolumn{3}{c}{ MUSCL } \\
$\mathrm{M}$ & $\left\|\epsilon_{\mathrm{Exp}}\right\|_{h}$ & $\left\|\epsilon_{\mathrm{Var}}\right\|_{h}$ & $\left\|\epsilon_{\mathrm{Exp}}\right\|_{h}$ & $\left\|\epsilon_{\mathrm{Var}}\right\|_{h}$ \\
\hline 1 & 0.1428 & 0.1897 & 0.1518 & 0.2007 \\
2 & 0.1336 & 0.1884 & 0.1614 & 0.1965 \\
3 & 0.0263 & 0.0854 & 0.0742 & 0.2102 \\
4 & 0.0330 & 0.0758 & 0.1079 & 0.2327 \\
5 & 0.0198 & 0.0407 & 0.0783 & 0.1887 \\
6 & 0.0176 & 0.0529 & 0.0733 & 0.1215 \\
7 & 0.0267 & 0.0813 & 0.0694 & 0.1419 \\
\hline
\end{tabular}
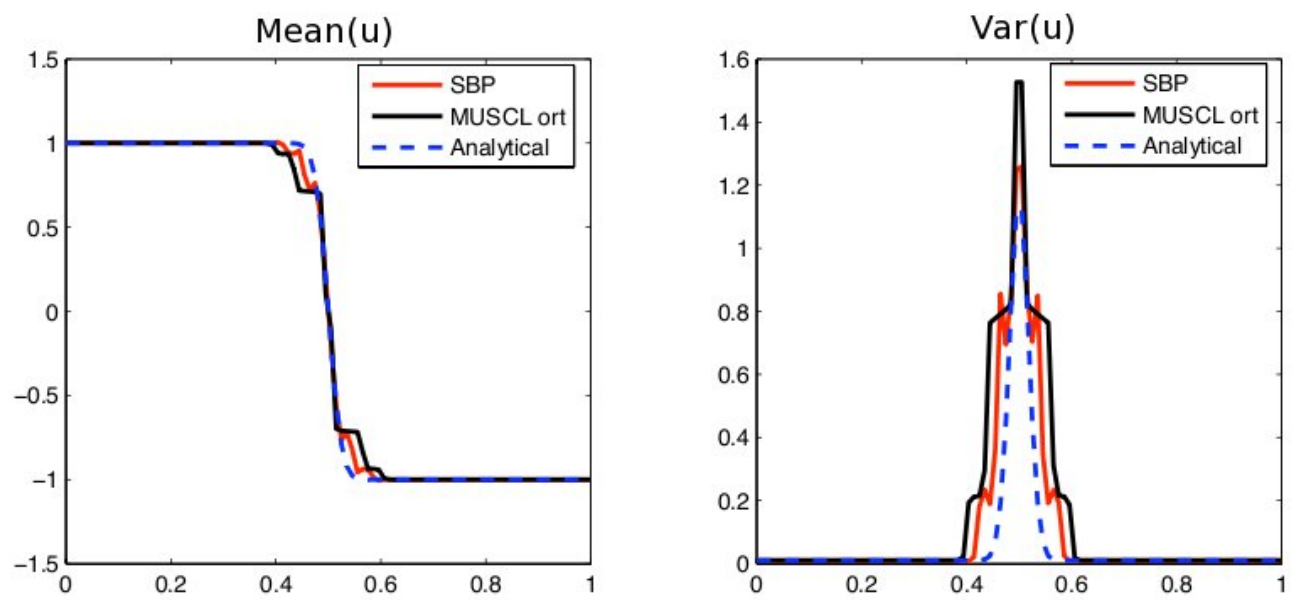

Figure 1: Comparison SBP and MUSCL. Locally weighted dissipation. $M=3, m=100, T=0.2$. Orthonormal Hermite polynomials.
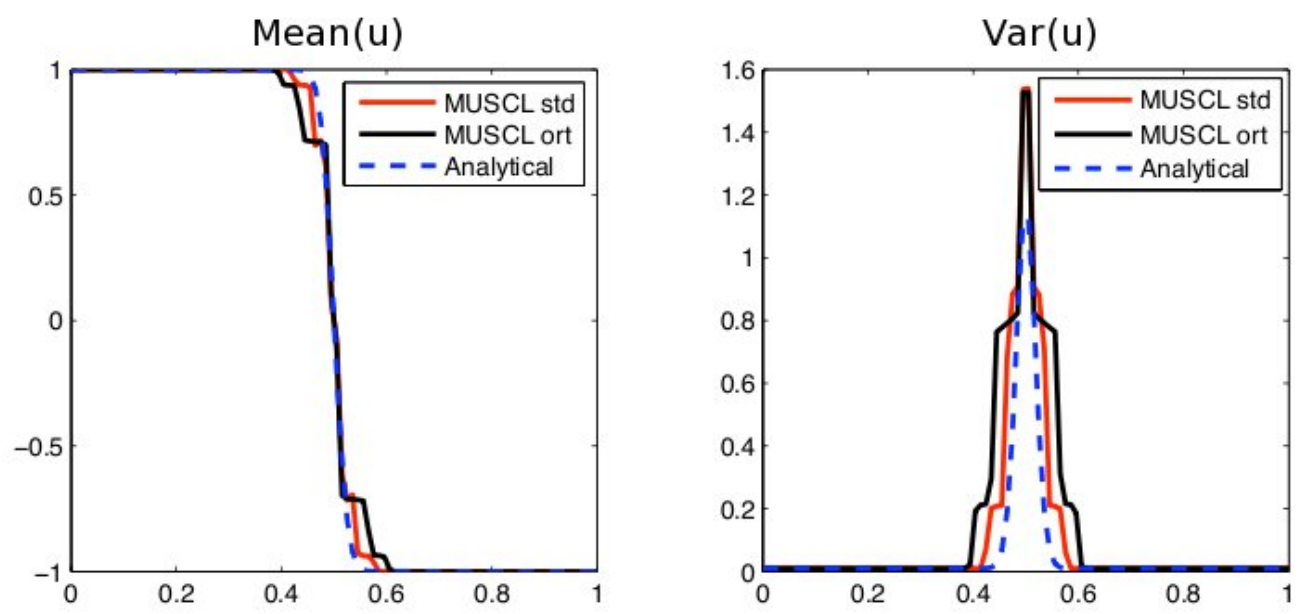

Figure 2: $M=3, m=100, T=0.2$. The label "Std" denotes a solution based on the standard probabilistic Hermite polynomials and "ort" denotes the scaling to make these polynomials orthonormal. 

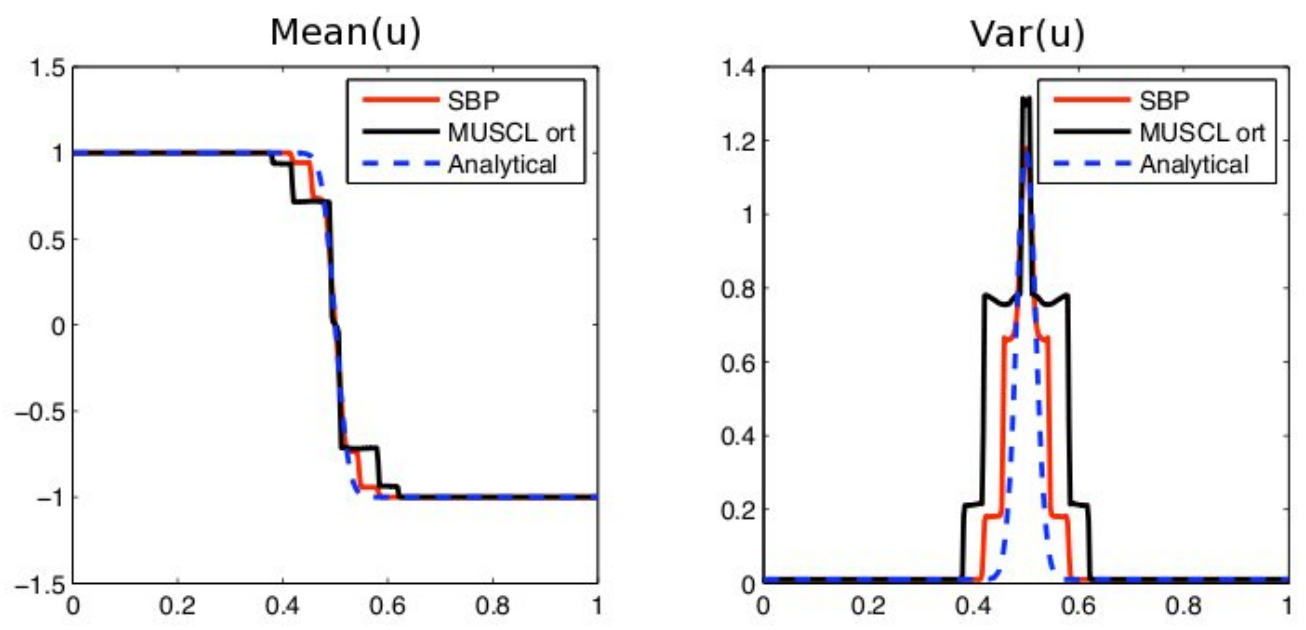

Figure 3: Comparison SBP and MUSCL. Dissipation based on largest eigenvalues. $M=3, m=400$, $T=0.2$.
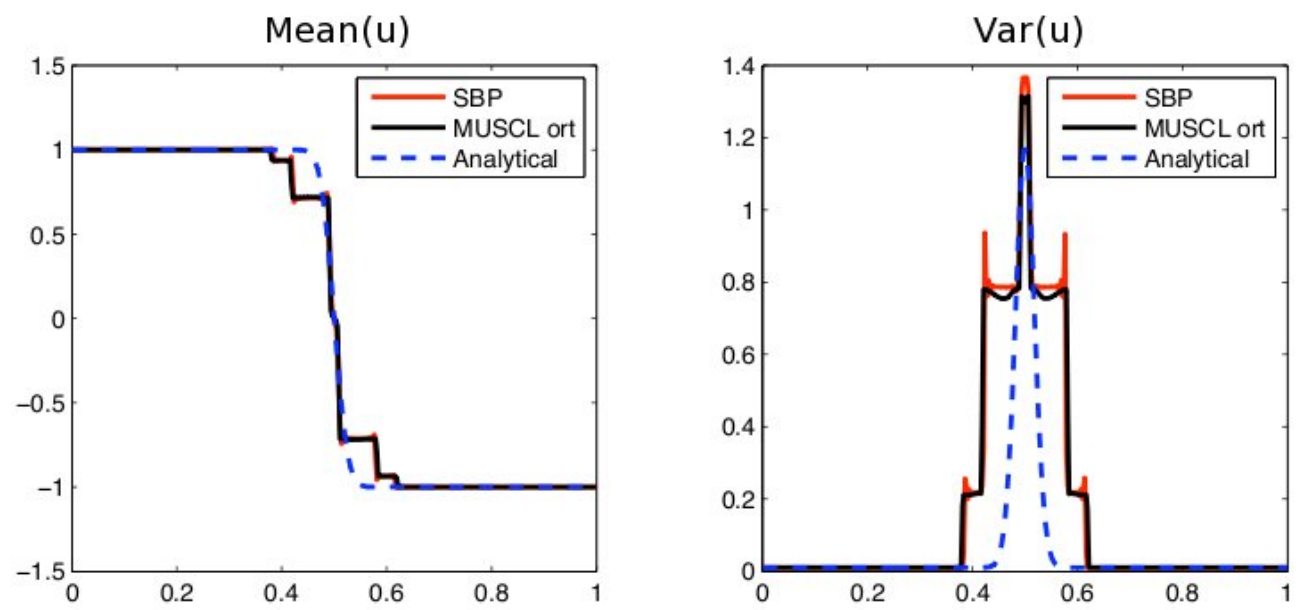

Figure 4: Comparison SBP and MUSCL. Locally weighted dissipation. $M=3, m=400, T=0.2$. 

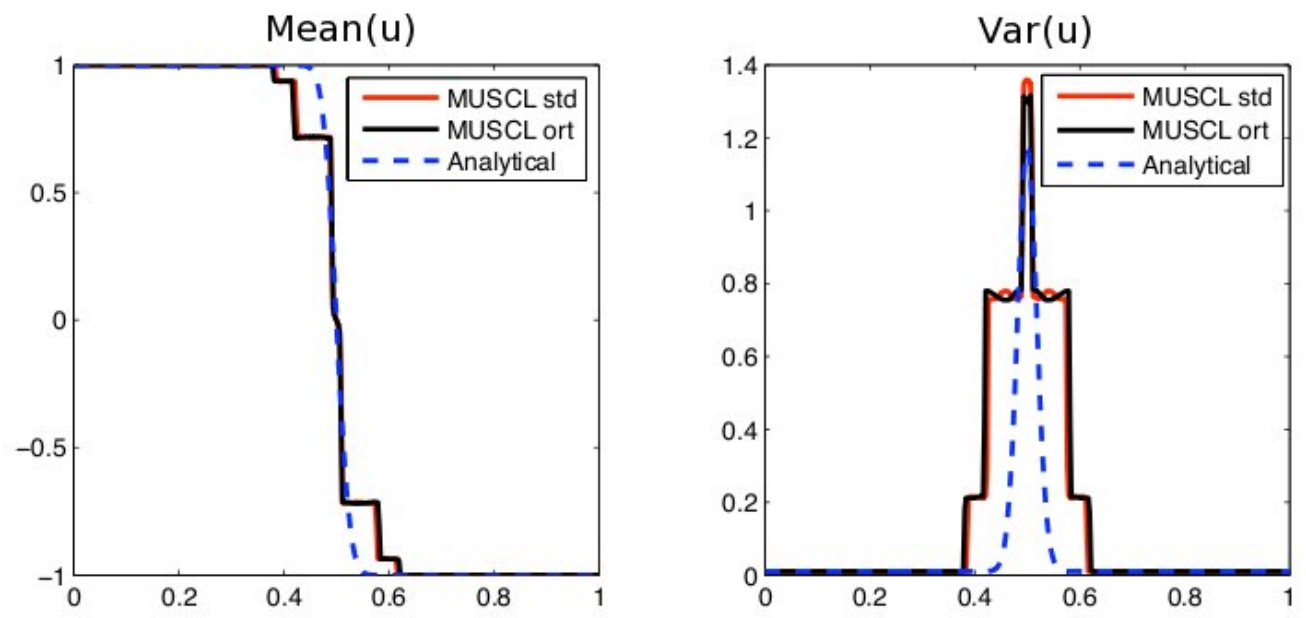

Figure 5: $M=3, m=400, T=0.2$. Standard basis and orthogonal Hermite basis.

This illustrates the fact that excessive use of artificial dissipation on a coarse mesh might appear to provide a more accurate solution to the original problem than the most accurate solution to the truncated system, given by the MUSCL scheme. This suggests that the effect of the truncation of the polynomial chaos expansion should be taken into account in the solution method.

\section{Conclusions}

Compared to the classical deterministic Burgers' equation where shocks are accurately captured by both the SBP method and the MUSCL scheme, high order polynomial chaos systems representing uncertain Burgers' equation are very sensitive to the choice of numerical method. The inexact estimate of the artificial dissipation for the central summation by parts operators often result in either oscillatory and eventually unstable schemes or inaccurate schemes with poor shock capturing properties. Therefore, the MUSCL scheme seems to be a more suitable choice of numerical method for these problems. However, the increasing number of shocks that result from higher order polynomial chaos requires finer grids and are therefore computationally expensive. Even with the MUSCL scheme, a different scaling of the basis polynomials affects the grid convergence.

\section{References}

[1] R. Ghanem and P. Spanos. Stochastic Finite Elements: A Spectral Approach. SpringerVerlag, 1991.

[2] M. H. Carpenter, J. Nordström, and D. Gottlieb. A stable and conservative interface treatment of arbitrary spatial accuracy. J. Comput. Phys., 148(2):341-365, 1999.

[3] M. H. Carpenter, D. Gottlieb, and S. Abarbanel. Time-stable boundary conditions for finitedifference schemes solving hyperbolic systems: methodology and application to high-order compact schemes. Journal of Computational Physics, 111:220-236, 1994.

[4] B. van Leer. Towards the ultimate conservative difference scheme. Journal of Computational Physics, 135:229-248, 1997. 
[5] R. LeVeque. Finite Volume Methods for Hyperbolic Problems. Cambridge University Press, 2002.

[6] P. Pettersson, G. Iaccarino, and J. Nordström. Numerical analysis of the Burgers' equation in the presence of uncertainty. Journal of Computational Physics, 228:8394-8412, 2009.

[7] K. Mattsson, M. Svärd, and J. Nordström. Stable and accurate artificial dissipation. J. Sci. Comput., 21(1):57-79, 2004.

[8] B. Strand. Summation by parts for finite difference approximations for d/dx. Journal of Computational Physics, 110(1):47-67, 1994.

[9] R. D. Richtmyer and K. V. Morton. Difference Methods for Initial-Value Problems. Interscience Publishers, 1967. 